\title{
INHOMOGENEOUS MINIMA OF A CLASS OF TERNARY QUADRATIC FORMS
}

\author{
MADHU RAKA
}

(Received 28 February 1991)

Communicated by J. H. Loxton

\begin{abstract}
Let $\Gamma_{2,1}^{(k)}$ denote the $k$ th successive inhomogeneous minima for positive values of real indefinite ternary quadratic forms of type $(2,1)$. Here it is proved that for the class of zero forms

$$
\Gamma_{2,1}^{(2)}=8 / 3, \quad \Gamma_{2,1}^{(3)}=9 / 4, \quad \Gamma_{2,1}^{(4)}=2
$$

All the critical forms have also been obtained. $\Gamma_{2,1}^{(1)}=4$ is already known. For non-zero forms it is proved that $\Gamma_{2,1}^{(1)}<10 / 3$.

1991 Mathematics subject classification (Amer. Math. Soc.): 10 E 20.
\end{abstract}

\section{Introduction}

Let $Q\left(x_{1}, x_{2}, \ldots, x_{n}\right)$ be a real indefinite quadratic form of determinant $D \neq 0$ and of type $(r, s), n=r+s$. Let $\Gamma_{r, s}$ be the infimum of real numbers $\Gamma$ for which the inequality

$$
0<Q\left(x_{1}+c_{1}, \ldots, x_{n}+c_{n}\right) \leq(\Gamma|D|)^{1 / n}
$$

is solvable in integers $x_{1}, \ldots, x_{n}$, for any real numbers $c_{1}, \ldots, c_{n}$ and for all quadratic forms $Q$ of type $(r, s)$. Similarly the symmetric non-homogeneous minimum $C_{r, s}$ is defined for which

$$
\left|Q\left(x_{1}+c_{1}, \ldots, x_{n}+c_{n}\right)\right| \leq\left(C_{r, s}|D|\right)^{1 / n}
$$

(C) 1993 Australian Mathematical Society 0263-6115/93 \$A2.00+0.00 
is solvable in integers $x_{1}, \ldots, x_{n}$.

The values of $\Gamma_{r, s}$ and $C_{r, s}$ are known for various $n$. See for reference Bambah, Dumir and Hans-Gill (1981) and Raka (1983). Davenport (1946) showed that $C_{1,1}$ and $\Gamma_{1,1}$ are not isolated. For $n=3$, Davenport (1948) showed that $C_{2,1}$ is isolated and later on Barnes $(1954,1956)$ obtained the second and third minima also for symmetric non-homogeneous ternary forms. In fact for $n \geq 3$, Vulakh (1985) has shown that $\Gamma_{r, s}$ and $C_{r, s}$ are isolated for rational forms. Whereas for irrational forms, it is known that (1.1) or (1.2) is solvable with arbitrary small constant, in view of Oppenheim's conjecture proved by Margulis (1987).

In this paper, we will obtain the second, third and fourth minima (namely $8 / 3,9 / 4$ and 2 ) for positive values of zero ternary quadratic forms of type $(2,1)$, (Theorem 1). The first minimum $\Gamma_{2,1}=4$ has been obtained by Barnes (1961) and Blaney (1950a) independently. For nonzero forms of type $(2,1)$, we prove that $\Gamma_{2,1}<\frac{10}{3}$ (Theorem 2) which is better than the above result of Barnes and Blaney. For the ternary forms of type $(1,2)$, Dumir and Hans-Gill (to appear) have shown that the second minima for positive values is $27 / 4$. In fact they showed that this is also isolated. The second minima for positive values of quinary forms of type $(2,3)$ has also been obtained by Bambah, Dumir and Hans-Gill (1984).

We prove the following results:

THEOREM 1. Let $Q(x, y, z)$ be a zero ternary quadratic form of type $(2,1)$ and determinant $D \neq 0$. For any real numbers $\left(x_{0}, y_{0}, z_{0}\right)$, we can find $(x, y, z) \equiv$ $\left(x_{0}, y_{0}, z_{0}\right) \quad(\bmod 1)$ satisfying

$$
0<Q(x, y, z)<(2|D|)^{1 / 3}
$$

except when $Q \sim \rho Q_{i}, i=1,2, \ldots, 8, \rho>0$. And for $Q_{i},(1.3)$ is soluble unless $\left(x_{0}, y_{0}, z_{0}\right) \equiv\left(x_{0}^{(i)}, y_{0}^{(i)}, z_{0}^{(i)}\right) \quad(\bmod 1)$ where $Q_{i}$ and $\left(x_{0}^{(i)}, y_{0}^{(i)}, z_{0}^{(i)}\right)$ are given in the following table:

\begin{tabular}{l|l|l|l}
1 & \multicolumn{1}{|c|}{$Q_{i}$} & $\left(x_{0}^{(i)}, y_{0}^{(i)}, z_{0}^{(i)}\right)$ & $\Gamma_{2,1}\left(Q_{i}\right)$ \\
\hline 1 & $x y+2 z^{2}$ & $(0,0,0)$ & 2 \\
2 & $\left(x+\frac{1}{2} y\right) y+2 z^{2}$ & $\left(\frac{1}{2}, 0,0\right)$ & 2 \\
3 & $4 x y+3 y^{2}+z^{2}$ & $\left(\frac{1}{2}, \frac{1}{2}, \frac{1}{2}\right)$ & 2 \\
4 & $4 x y+3 z^{2}$ & $(0,0,0) 4$ & $9 / 4$ \\
5 & $4\left(x+\frac{1}{2} y\right) y+3 z^{2}$ & $\left(\frac{1}{2}, 0,0\right)$ & $9 / 4$ \\
6 & $2 x y+y^{2}+y z+3 z^{2}$ & $\left(\frac{1}{2}, 0,0\right)$ & $8 / 3$ \\
7 & $x y+z^{2}$ & $(0,0,0)$ & 4 \\
8 & $\left(x+\frac{1}{2} y\right) y+z^{2}$ & $\left(\frac{1}{2}, 0,0\right)$ & 4
\end{tabular}


For the exceptional form $Q_{i}$, the inhomogeneous minimum $\Gamma_{2,1}\left(Q_{i}\right)$ is as shown in the table. Clearly the forms $Q_{i}$ are not equivalent.

THEOREM 2. Let $Q(x, y, z)$ be a non zero ternary quadratic form of type $(2,1)$ and determinant $D<0$. For any real numbers $\left(x_{0}, y_{0}, z_{0}\right)$, there exist $(x, y, z) \equiv\left(x_{0}, y_{0}, z_{0}\right) \quad(\bmod 1)$ such that

$$
0<Q(x, y, z)<(10|D| / 3)^{1 / 3} .
$$

\section{Some lemmas}

Let $\alpha, \beta(\alpha<\beta)$ be any real numbers. We say that the inequality

$$
\alpha<Q\left(x_{1}, \ldots, x_{n}\right)<\beta
$$

is soluble, if for any real numbers $c_{1}, \ldots, c_{n}$ there exist $\left(x_{1}, \ldots, x_{n}\right) \equiv$ $\left(c_{1}, \ldots, c_{n}\right) \quad(\bmod 1)$ satisfying $(2.1)$.

LEMMA 1. Let $\alpha, \beta, \gamma$ be real numbers with $\gamma>1$. Let $m$ be the integer such that $m<\gamma \leq m+1$. Then given any real number $x_{0}$, there exist $x \equiv x_{0}$ $(\bmod 1)$ such that

$$
0<(x+\alpha)^{2}+\beta<\gamma
$$

provided that

$$
-m^{2} / 4<\beta<\gamma-1 / 4 .
$$

This follows from Lemma 6 of Dumir (1968).

LEMMA 2. Let $\alpha, \beta, \gamma$ and $m$ be as above. Then

$$
0<-(x+\alpha)^{2}+\beta<\gamma
$$

is soluble in $x \equiv x_{0} \quad(\bmod 1)$, provided that

$$
1 / 4<\beta<\gamma+m^{2} / 4 .
$$

This follows from Lemma 2 of Dumir (1967). 
LEMMA 3. Let $\varphi(y, z)$ be an indefinite binary quadratic form of discriminant $\Delta$. Let $v$ be any positive real number. Then for any real numbers $y_{0}, z_{0}$, there exist $(y, z) \equiv\left(y_{0}, z_{0}\right) \quad(\bmod 1)$ satisfying

$$
-\Delta / 4 v<\varphi(y, z) \leq v \Delta / 4 .
$$

If $v \geq 3$, the following better inequality is soluble

$$
\frac{-\Delta}{\sqrt{(v+1)(\nu+9)}}<\varphi(y, z) \leq \frac{\nu \Delta}{\sqrt{(\nu+1)(v+9)}} .
$$

This is Theorem 1 and 2 of Blaney (1950b).

LEMMA 4. Let $\varphi(y, z)$ be as in Lemma 3, and $v \geq 4$. Then the following inequality

$$
-\Delta / 4 \sqrt{5}<\varphi(y, z) \leq \nu \Delta / 4 \sqrt{5}
$$

is soluble.

This follows from Theorem 2 of Grover and Raka (1991).

LEMMA 5. Let $\alpha$ and $A$ be positive numbers. Let $\beta$ be any real number. Let $2 h, k$ be integers such that

$$
\left|h \alpha^{3}-k^{2}\right|+\alpha^{3} / 2<A .
$$

Suppose that either

$$
\alpha^{3} \neq k^{2} / h \quad \text { or } \quad \beta \not \equiv \alpha h / k \quad\left(\bmod \alpha / k, 2 / \alpha^{2}\right)
$$

(that is, $\beta-\alpha h / k$ is not an integral combination of $\alpha / k$ and $\left.2 / \alpha^{2}\right)$. Then for any real number $v$, there exist integers $x, y$ satisfying

$$
0<v+\alpha x+\beta y \pm y^{2} / \alpha^{2}<A / \alpha^{2} \text {. }
$$

for any sign.

This is a result of Macbeath (1951), stated as Lemma 10 and its subsequent remarks in Bambah, Dumir and Hans-Gill (1984). 
LEMMA 6. Let $Q(x, y, z)$ be as in Theorem 1. Let $d=(2|D|)^{1 / 3}$. Suppose $Q$ represents $a$ value $\mu$ where either

$$
\begin{gathered}
0<\mu<d / 3, \quad \text { or } \\
d / 2.65 \leq \mu<d / 2, \quad \text { or } \\
d /(2.5)^{1 / 3}<\mu<d,
\end{gathered}
$$

then (1.3) is soluble.

PROOF. We can suppose, that

$$
Q(x, y, z)=\mu(x+h y+g z)^{2}+\varphi(y, z)
$$

where $\varphi(y, z)$ is an indefinite binary quadratic form of discriminant $\Delta^{2}=$ $4|D| / \mu^{2}$. By homogeneity we can assume that $\mu=1$.

Let $n<d \leq n+1$, so that $n \geq 1$. By Lemma 1 , it is enough to solve

$$
-n^{2} / 4<\varphi(y, z)<d-1 / 4 \text {. }
$$

Applying Lemma 3 with $v=\Delta / n^{2}>0$, we obtain

$$
-n^{2} / 4=-\Delta / 4 v<\varphi(y, z)<v \Delta / 4=2 d^{3} / 4 n^{2} .
$$

Then (2.10) will be satisfied if

$$
2 d^{3} /(4 d-1)<n^{2} .
$$

For $n \geq 3,(2.11)$ is easily seen to be true, since $d \leq n+1$. For $n=2,(2.11)$ is true if $d^{3}-8 d+2<0$ which certainly holds for $2<d \leq 2.65$. Thus (2.11) and hence (2.10) is true in case (2.7) or (2.8) holds. When (2.9) holds, we have $1<d<(2.5)^{1 / 3}$ and $n=1$. Apply Lemma 3 with $v=4 d-1 \geq 3$ to get (2.3). Then (2.10) will be satisfied if

$$
v \Delta / \sqrt{(v+1)(v+9)}<d-1 / 4 .
$$

Substituting for $v$ and $\Delta$ and simplifying, we see that this is so if

$$
2 d^{2}-(d+2)<0
$$

which is true for $d<(1+\sqrt{17}) / 4=1.28 \ldots$ If $1.28 . .<d<(2.5)^{1 / 3}$, apply Lemma 4 with $v=4 d-1>4$ to get

$$
-\Delta / 4 \sqrt{5}<\varphi(y, z) \leq \nu \Delta / 4 \sqrt{5}<(4 d-1) / 4 .
$$

This proves (2.10) and hence the lemma. 


\section{Proof of Theorem 1}

For zero form $Q$, we proceed as in Lemma 7 of Davenport (1948) and can assume that

$$
Q(x, y, z)=\lambda\left(x+\theta y+\theta^{\prime} z\right) y+z^{2}
$$

where $\lambda, \theta$ and $\theta^{\prime}$ are real numbers such that

$$
\begin{gathered}
\lambda>0 \quad \text { and } \quad-1 / 2<\theta^{\prime} \leq 1 / 2 . \\
\text { Let } \quad d=(2|D|)^{1 / 3}=\left(\lambda^{2} / 2\right)^{1 / 3} .
\end{gathered}
$$

LEMMA 7. If $d>3$, then (1.3) is soluble.

Proof follows from Lemma 6, (2.7), as $Q$ represents a value $\mu=1$.

LEMMA 8. If $2<d \leq 3$ then again (1.3) is soluble.

PROOF. If $y_{0} \equiv 0 \quad(\bmod 1)$, take $y=0$ and choose $z \equiv z_{0} \quad(\bmod 1)$ such that $0<z \leq 1$, so that

$$
0<Q(x, y, z)=z^{2} \leq 1<d .
$$

If $y_{0} \not \equiv 0 \quad(\bmod 1)$, choose $y$ such that $0<|y| \leq 1 / 2$. (3.3) gives $4<\lambda \leq$ $\sqrt{54}=7.348 \ldots$. For this $\lambda$, one can easily check that

$$
|\lambda| y|/ 2-1|+\lambda|y| / 2<d=\left(\lambda^{2} / 2\right)^{1 / 3} .
$$

Therefore the condition (2.5) of Lemma 5 is satisfied with

$$
\alpha^{3}=\lambda|y|, \quad d=A, \quad h=1 / 2 \quad \text { and } \quad k=1 .
$$

We see that (1.3) is soluble if

$$
0<(\operatorname{sign} y) \alpha^{3}\left(x+x_{0}+\theta y+\theta^{\prime}\left(z+z_{0}\right)\right)+\left(z+z_{0}\right)^{2}<d
$$

has a solution in integers $x$ and $z$.

If $y>0,(3.4)$ reduces to solving

$$
0<\alpha x+\beta z+z^{2} / \alpha^{2}+v<d / \alpha^{2}
$$

in integers $x$ and $z$ for some real numbers $\beta$ and $v$. By Lemma 5, (3.5) has a solution unless $\alpha^{3}=k^{2} / h=2$. And in that case, take $z$ arbitrary, and choose an integer $x$ such that

$$
0<\alpha x+\beta z+z^{2} / \alpha^{2}+v \leq \alpha<d / \alpha^{2} .
$$


If $y<0,(3.4)$ reduces to solving

$$
\begin{aligned}
-d / \alpha^{2} & <\alpha x+\beta^{\prime} z-z^{2} / \alpha^{2}+v^{\prime}<0, \quad \text { that is, } \\
0 & <\alpha x+\beta^{\prime} z-z^{2} / \alpha^{2}+v^{\prime \prime}<d / \alpha^{2}
\end{aligned}
$$

in integers $x$ and $z$, for some real numbers $\beta^{\prime}, v^{\prime}$ and $v^{\prime \prime}$. This can be solved again, using Lemma 5.

LEMMA 9. If $d \leq 2$ and $y_{0} \not \equiv 0 \quad(\bmod 1)$, then $(1.3)$ is soluble except when $Q \sim Q_{3}$ and $\left(x_{0}, y_{0}, z_{0}\right) \equiv(1 / 2,1 / 2,1 / 2) \quad(\bmod 1)$.

Proof. Choose $y \equiv y_{0} \quad(\bmod 1)$ such that $0<|y| \leq 1 / 2, z \equiv z_{0} \quad(\bmod 1)$ arbitrarily and $x \equiv x_{0} \quad(\bmod 1)$ to satisfy

$$
0<Q=\lambda\left(x+\theta y+\theta^{\prime} z\right) y+z^{2} \leq \lambda|y| \leq \lambda / 2 \leq d=\left(\lambda^{2} / 2\right)^{1 / 3}
$$

as $\lambda=\sqrt{2 d^{3}} \leq 4$. Therefore (1.3) is soluble with strict inequality unless $d=2$, $\lambda=4$ and $y_{0} \equiv 1 / 2 \quad(\bmod 1)$. In that case take $y=1 / 2$. Then $(1.3)$ is soluble if

$$
0<2\left(x+x_{0}+\theta / 2+\theta^{\prime}\left(z+z_{0}\right)\right)+\left(z+z_{0}\right)^{2}<2=d
$$

that is, if

$$
0<\alpha x+\beta z+z^{2} / \alpha^{2}+v<2 / \alpha^{2}
$$

has a solution in integers $x$ and $z$ where $\alpha^{3}=2, \beta=2\left(z_{0}+\theta^{\prime}\right) / \alpha^{2}$ and $v$ some real number.

Apply Lemma 5 with $h=1 / 2$ and $k=1$ to see that

$$
\left|h \alpha^{3}-k^{2}\right|+\alpha^{3} / 2=1<d=2 .
$$

Therefore (3.7) is solvable unless $\beta \equiv \alpha h / k \quad\left(\bmod \alpha / k, 2 / \alpha^{2}\right)$, that is, unless

$$
2 z_{0}+2 \theta^{\prime} \equiv 1 \quad(\bmod 2) \text {. }
$$

Similarly taking $y=-1 / 2$ and working as above, we see that (1.3) is soluble unless

$$
-2 z_{0}+2 \theta^{\prime} \equiv 1 \quad(\bmod 2) .
$$

From (3.8), (3.9) and (3.2) we get that

$$
\theta^{\prime}=0 \quad \text { or } \quad \theta^{\prime}=1 / 2 .
$$


When $\theta^{\prime}=0$, we have, from $(3.8), z_{0} \equiv 0$ or $1 / 2 \quad(\bmod 1)$. We discuss the case $\theta^{\prime}=0$ and $z_{0} \equiv 1 / 2 \quad(\bmod 1)$ only. The other cases are similar and are left to the reader. (The case $\theta^{\prime}=1 / 2$ and $z_{0} \equiv 0 \quad(\bmod 1)$ leads to the form $4 x y+2 y z+z^{2}$ and $x_{0} \equiv 0 \quad(\bmod 1)$, which is equivalent to $Q_{3}$ by the transformation $x \rightarrow x+y, z \rightarrow z-y$.) We have

$$
Q(x, y, z)=4(x+\theta y) y+z^{2} .
$$

We can assume (applying a suitable unimodular transformation) that $-1 / 4<$ $\theta \leq 3 / 4$.

Choose $x \equiv x_{0} \quad(\bmod 1)$ such that $0 \leq|x| \leq 1 / 2$, take $y=(\operatorname{sign} x) / 2$ and $z=1 / 2$, so that

$$
0<Q=2|x|+\theta+1 / 4 \leq 1+3 / 4+1 / 4=d
$$

Thus (1.3) is soluble unless $\theta=3 / 4, x_{0} \equiv 1 / 2 \quad(\bmod 1)$, that is,

$$
Q=Q_{3}=4 x y+3 y^{2}+z^{2} \quad \text { and } \quad\left(x_{0}, y_{0}, z_{0}\right) \equiv(1 / 2,1 / 2,1 / 2) \quad(\bmod 1) .
$$

LEMMA 10. If $d \leq 2$ and $y_{0} \equiv 0 \quad(\bmod 1)$, then $(1.3)$ is soluble except when $z_{0} \equiv 0 \quad(\bmod 1)$ and
(i) $\quad \lambda=1 / 2, \theta^{\prime}=0$
(ii) $\lambda=2 / 3, \theta^{\prime}=1 / 2$
(iii) $\lambda=1, \theta^{\prime}=0$
(iv) $\lambda=4 / 3, \theta^{\prime}=0$ or $1 / 2$.

PROOF. If $d>1$, take $y=0$, choose $x \equiv x_{0} \quad(\bmod 1)$ arbitrarily and $z \equiv z_{0}$ $(\bmod 1)$ such that $0<z \leq 1$. So that

$$
0<Q=\lambda\left(x+\theta y+\theta^{\prime} z\right) y+z^{2}=z^{2} \leq 1<d .
$$

If $d<1 / 2$, take $y=1$, choose $z \equiv z_{0} \quad(\bmod 1)$ arbitrarily and $x \equiv x_{0}$ (mod 1) such that

$$
0<Q=\lambda\left(x+\theta+\theta^{\prime} z\right)+z^{2} \leq \lambda=\sqrt{2 d^{3}}<d .
$$

Thus we need consider $1 / 2 \leq d \leq 1$ only which gives $1 / 2 \leq \lambda \leq \sqrt{2}$. Also if $z_{0} \not \equiv 0 \quad(\bmod 1)$, take $y=0, x \equiv x_{0} \quad(\bmod 1)$ arbitrarily and $z \equiv z_{0}$ $(\bmod 1)$ such that $0<|z| \leq 1 / 2$ so that

$$
0<Q(x, y, z)=z^{2} \leq 1 / 4<d .
$$


Therefore we must have $z_{0} \equiv 0 \quad(\bmod 1)$. Now take $y=1$ and $\alpha^{3}=\lambda$. Then (1.3) is soluble if

$$
0<\alpha x+\beta x+z^{2} / \alpha^{2}+v<d / \alpha^{2}
$$

has a solution in integers $x$ and $z$, where $\beta=\alpha \theta^{\prime}$ and $\nu=\alpha\left(x_{0}+\theta\right)$.

Take

$$
\begin{aligned}
& h=2 \quad \text { and } \quad k=1 \quad \text { if } 1 / 2 \leq \lambda \leq 3 / 5 \text {, } \\
& h=3 / 2 \text { and } k=1 \quad \text { if } 3 / 5<\lambda \leq 4 / 5 \text {, } \\
& h=1 \quad \text { and } k=1 \quad \text { if } 4 / 5<\lambda \leq 5 / 4 \text {, } \\
& h=3 \quad \text { and } k=2 \quad \text { if } 5 / 4<\lambda \leq \sqrt{2} \text {. }
\end{aligned}
$$

One can easily check that

$$
\left|h \lambda-k^{2}\right|+\lambda / 2<d=\left(\lambda^{2} / 2\right)^{1 / 3} .
$$

Now apply Lemma 5 , with $A=d$ and $\alpha^{3}=\lambda$, we see that (3.11) has a solution in integers $x$ and $z$ unless when

$$
\lambda=k^{2} / h \quad \text { and } \quad \theta^{\prime} \equiv h / k \quad(\bmod 1 / k, 2 / \lambda) .
$$

Since from (3.2), $-1 / 2<\theta^{\prime} \leq 1 / 2,(3.12)$ and (3.13) give the desired exceptional cases (3.10).

3.1. The exceptional cases. Firstly we note that (1.3) is soluble if the following inequality

(3.14) $0<F(x, y, z)=\frac{1}{\lambda} Q(x, y, z)<d / \lambda=(1 / 2 \lambda)^{1 / 3}=\delta \quad$ (say)

is soluble in $(x, y, z) \equiv\left(x_{0}, y_{0}, z_{0}\right) \quad(\bmod 1)$.

LEMMA 11. If $\lambda=1 / 2, \theta^{\prime}=0$ and $y_{0} \equiv 0 \equiv z_{0} \quad(\bmod 1)$ then (3.14) and hence (1.3) is soluble unless $2 Q=F \sim Q_{1}$ or $Q_{2}$ and $(x, y, z) \equiv\left(x_{0}^{(i)}, y_{0}^{(i)}, z_{0}^{(i)}\right)$ $(\bmod 1) ; i=1,2$.

PROOF. Here $F(x, y, z)=(x+\theta y) y+2 z^{2}$ and $\delta=1$. We can assume that $|\theta| \leq 1 / 2$.

Take $y=1, z=0$ and choose $x \equiv x_{0} \quad(\bmod 1)$ such that $0<x+\theta \leq 1$, so that

$$
0<F(x, 1,0)=x+\theta \leq 1=\delta .
$$


Thus (3.14) is soluble unless $x+\theta=1$, that is, unless

$$
x_{0}+\theta \equiv 0 \quad(\bmod 1) \text {. }
$$

Similarly taking $y=-1$ and $z=0$ we see that (3.14) is soluble unless

$$
x_{0}-\theta \equiv 0 \quad(\bmod 1) \text {. }
$$

From (3.15) and (3.16) we get either $\theta=0$ and $x_{0} \equiv 0 \quad(\bmod 1)$ or $\theta=1 / 2$ and $x_{0} \equiv 1 / 2 \quad(\bmod 1)$. This gives the exceptional forms $Q_{i}$ for $i=1$ and 2 .

LEMMA 12. Let $z_{0} \equiv 0 \quad(\bmod 1)$, and let the section of $F(x, y, z)$, namely $F(x, y, 0)=(x+\theta y) y$, represent a value $-v, v>0$, at a primitive point, satisfying:

$$
\begin{aligned}
& 0<v<1 / 2 \text { when } \lambda=2 / 3 \\
& 0<v<\delta / 4 \quad \text { or } \delta / 3.9 \leq v<1 / 2 \quad \text { when } \lambda=1 \\
& 0<v<\delta / 3 \quad \text { or } \delta / 2.81 \leq v<1 / 2 \quad \text { when } \lambda=4 / 3 .
\end{aligned}
$$

Then (3.14) is soluble.

PROOF. Applying a suitable unimodular transformation in which $z$ is transformed into itself, we can assume that

$$
F(x, y, z)=-v\left(x+a_{1} y+a_{2} z\right)^{2}+\frac{1}{4 v}\left(y+b_{2} z\right)^{2}+\frac{1}{\lambda} z^{2} .
$$

Let $m$ be an integer such that $m<\delta / v \leq m+1$. Then $m \geq 1$, since $\delta / v>2 \delta=2(1 / 2 \lambda)^{1 / 3}>1$. Now by Lemma $2,(3.14)$ is soluble if

$$
\frac{1}{4}<\frac{1}{4 v^{2}}\left(y+b_{2} z\right)^{2}+\frac{1}{\lambda v} z^{2}<\frac{m^{2}}{4}+\frac{\delta}{v},
$$

that is, if

$$
v^{2}<\psi(y, z)=\left(y+b_{2} z\right)^{2}+4 v z^{2} / \lambda<m^{2} v^{2}+4 \delta v
$$

is soluble in $(y, z) \equiv\left(y_{0}, 0\right)(\bmod 1)$. Take $z=1$ and choose $y \equiv y_{0}$ (mod 1) such that $0 \leq\left|y+b_{2}\right| \leq 1 / 2$, so that

$$
v^{2}<4 v / \lambda \leq \psi(y, z) \leq 1 / 4+4 v / \lambda .
$$


Then (3.20) will be satisfied if

$$
f(v)=m^{2} v^{2}+4 v(\delta-1 / \lambda)>1 / 4 .
$$

For $m \geq 3, f(v)$ is an increasing function of $v$ and $v \geq \delta /(m+1)$. Therefore,

$$
f(v) \geq f\left(\frac{\delta}{m+1}\right)=\delta^{2}\left(\frac{m+2}{m+1}\right)^{2}-\frac{4 \delta}{\lambda(m+1)}=g(m) \quad \text { (say) }
$$

Now $g(m)$ is an increasing function of $m$. One can verify that

$$
g(m)>1 / 4
$$

for $m \geq 6$ when $\lambda=2 / 3$; for $m \geq 4$ when $\lambda=1$; and for $m \geq 3$ when $\lambda=4 / 3$. In the remaining cases, take $z=0$ and choose $y \equiv y_{0} \quad(\bmod 1)$ such that $1 / 2 \leq|y| \leq 1$. Working as before one finds that (3.20) will be satisfied if

$$
f(v)=m^{2} v^{2}+4 \delta v>1 .
$$

Since $v \geq \delta /(m+1)$, this is so if

$$
f(\delta /(m+1))=(m+2)^{2} \delta^{2} /(m+1)^{2}>1 .
$$

For $\lambda=2 / 3$ and $m \leq 5, \lambda=1$ and $m \leq 2, \lambda=4 / 3$ and $m=1$, one checks that (3.23) is true. When $\lambda=1$ and $m=3$, we have $v \geq \delta /(3.9)$ from (3.18) and then

$$
f(v) \geq f(\delta / 3.9)=(24.6) \delta^{2} /(3.9)^{2}>1 .
$$

Similarly when $\lambda=4 / 3$ and $m=2$, we have $v \geq \delta / 2.81$ and (3.22) is satisfied. This proves the lemma.

LEMMA 13. If $\lambda=2 / 3, \theta^{\prime}=1 / 2$ and $y_{0} \equiv z_{0} \equiv 0 \quad(\bmod 1)$ then $(3.14)$ and hence $(1.3)$ is soluble unless $2 F=3 Q \sim Q_{6}$ and $x_{0} \equiv 1 / 2 \quad(\bmod 1)$.

PROOF. Here

$$
F(x, y, z)=(x+\theta y+z / 2) y+3 z^{2} / 2 .
$$

Suppose without loss of generality that $-1 / 2<\theta \leq 1 / 2$. If $-1 / 2<\theta<0$, then $F(x, y, 0)$ represents a value $\theta=-v$ with $0<v<1 / 2$, so the result follows from (3.17) of Lemma 12. Let now $0<\theta<1 / 2$. We have

$$
\frac{1}{\theta} F(x, y, z)=\left(y+\frac{x}{2 \theta}+\frac{z}{4 \theta}\right)^{2}+\frac{3}{2 \theta} z^{2}-\frac{1}{4 \theta^{2}}\left(x+\frac{1}{2} z\right)^{2} .
$$


Let $m$ be an integer $(\geq 1)$ such that $m<\delta / \theta \leq(m+1)$. (Note that $\delta / \theta=$ $\left.(3 / 4)^{1 / 3} / \theta>1\right)$. Then by Lemma $1,(3.14)$ is soluble if there exist $(x, z) \equiv$ $\left(x_{0}, 0\right) \quad(\bmod 1)$ such that

$$
-\frac{m^{2}}{4}<\frac{3}{2 \theta} z^{2}-\frac{1}{4 \theta^{2}}\left(x+\frac{1}{2} z\right)^{2}<\frac{\delta}{\theta}-\frac{1}{4} .
$$

Take $z=0$ and choose $x \equiv x_{0} \quad(\bmod 1)$ such that $0 \leq|x| \leq 1 / 2$. One finds that (3.25) is true for $m \geq 2$. If $m=1$, that is, when $\delta / 2 \leq \theta<1 / 2$, the section $F(x, y, 0)$ represents a value $-v=4 \theta-2$ at $(-1,2)$, where $0<v=2-4 \theta<$ $2-2 \delta<1 / 2$; then the result follows from (3.17) of Lemma 12 .

Thus we need consider $\theta=0$ and $1 / 2$ only. If $\theta=0$ and $x_{0} \equiv 0 \quad(\bmod 1)$, $(x, y, z)=(0,-2,1)$ gives a solution of $(3.14)$. If $x_{0} \neq \equiv 0 \quad(\bmod 1)$, we again have a solution of (3.14) by choosing $x$ such that $0<|x| \leq 1 / 2$ and taking $z=0, y=(\operatorname{sign} x) 1$. When $\theta=1 / 2$ and $x_{0} \not \equiv 1 / 2 \quad(\bmod 1)$, we get a solution of (3.14) by choosing $x$ such that $0 \leq|x|<1 / 2$ and taking $z=0, y=-(\operatorname{sign} x) 1$. When $\theta=1 / 2$ and $x_{0} \equiv 1 / 2 \quad(\bmod 1)$, we have the exceptional form $2 F=2 x y+y^{2}+y z+3 z^{2}=Q_{6}$.

LEMMA 14, then (3.14) and hence (1.3) is soluble unless $Q \sim Q_{7}$ and $x_{0} \equiv 0$ $(\bmod 1)$, or $Q \sim Q_{8}$ and $x_{0} \equiv 1 / 2 \quad(\bmod 1)$.

PROOF. Here

$$
Q=F(x, y, z)=(x+\theta y) y+z^{2} .
$$

Suppose without loss of generality that $-1 / 2<\theta \leq 1 / 2$. if $-\delta / 4<\theta<0$ or $-1 / 2<\theta<-\delta / 3.9$, then the section $F(x, y, 0)$ represents a value $\theta=-v$, and the result follows from (3.18) of Lemma 12. If $-\delta / 3.9 \leq \theta<-\delta / 4$, $Q(x, y, z)$ represents a value $\mu=4 \theta+1$ where $0<4 \theta+1<d / 3$. (Note that here $d=\delta=(1 / 2)^{1 / 3}$ ). So the result follows from (2.7) of Lemma 6 . If $0<\theta<d / 2$, we have

$$
\frac{1}{\theta} F(x, y, z)=\left(y+\frac{x}{2 \theta}\right)^{2}+\frac{z^{2}}{\theta}-\frac{x^{2}}{4 \theta^{2}} .
$$

Apply Lemma 1, and working as usual, one finds that (3.14) is soluble on choosing $z=0$ and $x \equiv x_{0} \quad(\bmod 1)$ such that $0 \leq|x| \leq 1 / 2$.

If $d / 2 \leq \theta<(d+1) / 4$, we have

$$
d /(2.5)^{1 / 3}<\mu=Q(-1,2,1)=4 \theta-1<d .
$$


If $(d+1) / 4 \leq \theta<(d / 3+7) / 16$, we have

$$
0<\mu=Q(-4,4,3)=16 \theta-7<d / 3 \text {. }
$$

Then the result follows from (2.7) and (2.8) of Lemma 6. If $(d / 3+7) / 16 \leq \theta<$ $1 / 2$, the Section $F(x, y, 0)$ represents a value $-v=4 \theta-2$ where $0<v<d / 4$, and the result follows from (3.18) of Lemma 12. Thus we are left with $\theta=0$ and $1 / 2$ only. This gives $Q=Q_{7}$ and $Q_{8}$. One easily finds that (1.3) is soluble for $Q_{7}$ or $Q_{8}$ except when $x_{0} \equiv 0$ or $1 / 2 \quad(\bmod 1)$ respectively.

LEMMA 15. If $\lambda=4 / 3$ and $\theta^{\prime}=0$, then (3.14) and hence (1.3) is soluble unless $\theta=0$ or $1 / 2$, that is, unless $4 F=3 Q \sim Q_{4}$ and $x_{0} \equiv 0(\bmod 1)$ or $3 Q \sim Q_{5}$ and $x_{0} \equiv 1 / 2 \quad(\bmod 1)$.

LEMMA 16. If $\lambda=4 / 3$ and $\theta^{\prime}=1 / 2$, then (3.14) and hence (1.3) is soluble unless $\theta=-1 / 4$ or $1 / 4$, that is, unless $3 Q \sim Q_{4}$ and $x_{0} \equiv 0(\bmod 1)$ or $3 Q \sim Q_{5}$ and $x_{0} \equiv 1 / 2 \quad(\bmod 1)$.

Proofs of Lemmas 15 and 16 are similar and are left to the reader. For the exceptional forms $Q_{i}$, and $\left(x_{0}, y_{0}, z_{0}\right) \equiv\left(x_{0}^{(i)}, y_{0}^{(i)}, z_{0}^{(i)}\right) \quad(\bmod 1), 1 \leq i \leq 8$, one can easily check that $\Gamma_{2,1}\left(Q_{i}\right)$ is as given in the table. This completes the proof of Theorem 1 .

\section{Proof of Theorem 2}

We need the following two results of Barnes (1955) and Cassels stated as Lemma 3 in Barnes (1954).

LEMMA 17. Let $Q(x, y, z)$ be as in Theorem 2. Then there exist integers $x_{1}$, $y_{1}, z_{1}$ such that

$$
0<Q\left(x_{1}, y_{1}, z_{1}\right) \leq(4|D| / 3)^{1 / 3}
$$

LEMMA 18. Let $U$ be a $2 \times 2$ unimodular matrix of infinite order and $\mathscr{R}$ be a bounded set in $\mathbb{R}^{2}$. Let $\mathscr{R}$ have the property.

$$
\begin{array}{cl}
U(\mathscr{R}) \cap(\mathscr{R}+A) \neq \emptyset & \text { for some } A \in \mathbb{Z}^{2} \\
\text { but } \quad U(\mathscr{R}) \cap(\mathscr{R}+B)=\emptyset, & \forall B \in \mathbb{Z}^{2}, B \neq A .
\end{array}
$$

If $P$ is a point such that $U^{n}(P)$, for all integers $n \equiv 0$, is congruent $(\bmod 1)$ to a point of $\mathscr{R}$, then $P$ is the unique fixed point given by $U(P)-A=P$. 
Using Lemma 17, and working as usual, see for example Dumir (1968), we see that it suffices to prove

THEOREM A. Let $Q(x, y, z)=(x+h y+g z)^{2}+\phi(y, z)$, where $\phi(y, z)$ is a non-zero indefinite binary quadratic form of discriminant

$$
\begin{gathered}
\Delta^{2}=4|D| \geq 3, \quad \text { and } \\
|h| \leq 1 / 2, \quad|g| \leq 1 / 2 .
\end{gathered}
$$

Suppose further that for integers $x, y, z$ we have

$$
\text { either } \quad Q(x, y, z) \leq 0 \quad \text { or } \quad Q(x, y, z) \geq 1-\varepsilon
$$

where $\varepsilon(\geq 0)$ is sufficiently small. Let

$$
d=(10|D| / 3)^{1 / 3} .
$$

Then there exist $(x, y, z) \equiv\left(x_{0}, y_{0}, z_{0}\right) \quad(\bmod 1)$ satisfying

$$
0<Q(x, y, z)<d \text {. }
$$

LEMMA 19. If $Q(x, y, z)$ is as in Theorem $A$, then for integers $y, z$ we have either

$$
\phi(y, z)=0 \quad \text { or } \phi(y, z) \leq-1 / 4 \quad \text { or } \phi(y, z) \geq 3 / 4-\varepsilon .
$$

For proof see Dumir (1968).

PROOF OF THEOREM A. From (4.2) and (4.5) we get $d \geq(5 / 2)^{1 / 3}$. Let $n$ be an integer ( $\geq 1$ ) such that $n<d \leq n+1$. Then by Lemma 1 , it suffices to solve

$$
-n^{2} / 4<\phi(y, z)<d-1 / 4 \text {. }
$$

LEMMA 20. If $n \geq 2$, then (4.8) and hence (4.6) is soluble.

Proof. Apply Lemma 3 with $v=\Delta / n^{2}$ to get

$$
-n^{2} / 4=-\Delta / 4 v<\phi(y, z)<v \Delta / 4=\Delta^{2} / 4 n^{2} .
$$

Then $(4.8)$ will be satisfied if

$$
\Delta^{2} /(4 d-1)=6 d^{3} / 5(4 d-1)<n^{2} .
$$

This can be easily seen to be true for $d \leq n+1$ and $n \geq 2$. 
Let now $n=1,(5 / 2)^{1 / 3} \leq d \leq 2$. Let

$$
\begin{aligned}
& \phi_{1}=y^{2}+y z-z^{2} \\
& \phi_{2}=y^{2}-2 z^{2} \\
& \phi_{3}=5 y^{2}+11 y z-5 z^{2} .
\end{aligned}
$$

By the Markoff Chain Theorem, if $\phi \nsucc \rho \phi_{1}, \rho \phi_{2}$ and $\rho \phi_{3}$ then there exist integers $u, v$ not both zero with $(u, v)=1$ such that $a=\phi(u, v)$ and $|a| \leq 13 \Delta / \sqrt{1517}$. In fact one can suppose that $|a| \leq \Delta / 3$ unless $\phi$ is a Markoff form. A Markoff form represents both $a$ and $-a$, so for such forms we can assume that $a>0$. Since $\phi$ is a non-zero form, we distinguish the following cases using (4.7):

I. $3 / 4-\varepsilon \leq a \leq 13 \Delta / \sqrt{1517}$;

II. $a=-b<0,1 / 4 \leq b \leq \Delta / 3$, or $\phi(y, z) \sim a \phi_{1}$ or $a \phi_{3}, a=-b<0$;

III. $\phi(y, z) \sim \rho \phi_{2}, \rho>0$.

Replacing $\phi(y, z)$ by an equivalent form we can suppose that

$$
\phi(y, z)=a(y+f z)^{2}-\frac{\Delta^{2}}{4 a} z^{2} .
$$

LEMMA 21. If $3 / 4-\varepsilon \leq a \leq 13 \Delta / \sqrt{1517}$, then (4.8) and hence (4.6) is true.

PROOF. Choose $z \equiv z_{0} \quad(\bmod 1)$ such that $0 \leq|z| \leq 1 / 2$. If $0 \leq|z|<$ $\sqrt{\left(a^{2}+a\right)} / \Delta$, choose $y \equiv y_{0} \quad(\bmod 1)$ such that $1 / 2 \leq|y+f z| \leq 1$, so that

$$
-1 / 4=a / 4-\Delta^{2}\left(a^{2}+a\right) / 4 a \Delta^{2}<\phi(y, z) \leq a<d-1 / 4 .
$$

If $\sqrt{\left(a^{2}+a\right)} / \Delta \leq|z| \leq 1 / 2$ and $d / a>2$, choose $y \equiv y_{0} \quad(\bmod 1)$ such that $1 \leq|y+f z| \leq 3 / 2$ to get (noting that $a \geq \frac{3}{4}-\varepsilon$ and $\Delta^{2}<11$ ):

$$
-1 / 4<a-\Delta^{2} / 16 a \leq \phi(y, z) \leq q a / 4-(a+1) / 4=2 a-1 / 4<d-1 / 4 .
$$

If $\sqrt{\left(a^{2}+a\right)} / \Delta \leq|z| \leq 1 / 2$ and $d / a \leq 2$, shift $z$ by \pm 1 to get $1 / 2 \leq|z| \leq$ $1-\sqrt{\left(a^{2}+a\right)} / \Delta$.

Now choose $y \equiv y_{0} \quad(\bmod 1)$ such that $1 \leq|y+f z|<3 / 2$, so that

$$
a-\frac{\Delta^{2}}{4 a}\left\{1-\frac{1}{\Delta} \sqrt{a^{2}+a}\right\}^{2} \leq \phi(y, z) \leq \frac{9}{4} a-\frac{\Delta^{2}}{16 a} .
$$


Then (4.8) will be satisfied if

$$
\begin{aligned}
9 a^{2}-a(4 d-1) & <\Delta^{2} / 4 \\
\frac{\Delta^{2}}{4 a}\left\{1-\frac{1}{\Delta} \sqrt{a^{2}+a}\right\}^{2} & <a+\frac{1}{4} .
\end{aligned}
$$

One can easily check that (4.10) and (4.11) are true for

$$
d / 2 \leq a \leq 13 \Delta / \sqrt{1517}<\Delta / \sqrt{8.97} \text { and } d \leq 2
$$

LEMMA 22. If $a=-b, 1 / 4 \leq b \leq \Delta / 3$, then again (4.8) and hence (4.6) is true.

PROOF. (4.8) is equivalent to solving

$$
0<-(y+f z)^{2}+\Delta^{2} z^{2} / 4 b^{2}+1 / 4 b<d / b .
$$

Let $m$ be an integer such that $m<d / b \leq m+1$. Since $d \leq 2$, we have here $1 \leq m \leq 7$. By Lemma 2, it is enough to solve

$$
1 / 4<g(z)=\Delta^{2} z^{2} / 4 b^{2}+1 / 4 b<d / b+m^{2} / 4
$$

Choose $z \equiv z_{0} \quad(\bmod 1)$ such that $0 \leq|z| \leq 1 / 2$. One can easily check, for $b \geq d /(m+1)$ and $m \leq 7$, that the second inequality in (4.13) is satisfied. If $b<$ 1 the first inequality in (4.13) is also satisfied. If $b \geq 1$ but $|z|>\sqrt{\left(b^{2}-b\right)} / \Delta$, we still have $g(z)>1 / 4$, proving (4.13).

Let now $b \geq 1$ and $0 \leq|z| \leq \sqrt{\left(b^{2}-b\right)} / \Delta$. Shift $z$ by \pm 1 to get

$$
1 \leq|z| \leq 1+\sqrt{\left(b^{2}-b\right)} / \Delta \text {. }
$$

Then choose $y \equiv y_{0} \quad(\bmod 1)$ such that $1 \leq|y+f z| \leq 3 / 2$, so that

$$
-\frac{9}{4} b+\frac{\Delta^{2}}{4 b} \leq \phi(y, z) \leq-b+\frac{\Delta^{2}}{4 b}\left\{1+\frac{1}{\Delta} \sqrt{\left(b^{2}-b\right)}\right\}^{2} .
$$

Now (4.8) will be satisfied if

$$
\begin{gathered}
\Delta^{2} / 4 b^{2}+1 / 4 b>9 / 4 \\
-1+\Delta^{2}\left\{1+\sqrt{\left(b^{2}-b\right)} / \Delta\right\}^{2} / 4 b^{2}<(4 d-1) / 4 b .
\end{gathered}
$$


(4.14) follows immediately because $b \leq \Delta / 3$. (4.15) is true if on simplifying we have

$$
f(b)=3 b^{2}+4 b d-2 \Delta \sqrt{b^{2}-b}>\Delta^{2} .
$$

Since $b \leq \Delta / 3 \leq \sqrt{16 / 15}, \sqrt{b^{2}-b}<1 / 5$, we have

$$
f(b) \geq 3+4 d-2 \Delta / 5>\Delta^{2} \text { for } d \leq 2 .
$$

This proves (4.16) and hence the lemma.

LEMMA 23. If $\phi \sim-b \phi_{1}$ or $-b \phi_{3}$ then (4.8) and hence (4.6) is true.

PROOF. We can have

$$
\phi=-b(y+f z)^{2}+\Delta^{2} z^{2} / 4 b
$$

where $b=\Delta / \sqrt{5}$ or $5 \Delta / \sqrt{221}$ and $d / b=\sqrt{25 / 6 d}$ or $\sqrt{221 / 30 d}$ according as $\phi=-b \phi_{1}$ or $-b \phi_{3}$. We work as in Lemma 22 .

When $\phi=-b \phi_{1}$, we have $m=1$ only. Choose $z \equiv z_{0} \quad(\bmod 1)$ such that $1 / 2 \leq|z| \leq 1$. One can easily check that

$$
1 / 4<g(1 / 2) \leq g(z) \leq g(1)<d / b+1 / 4 .
$$

proving thereby (4.13) and hence (4.8).

When $\phi=-b \phi_{3}$, we have $m=1$ or 2 . Working exactly as in Lemma 22, we see that it is enough to prove (4.14) and (4.16) for $b=5 \Delta / \sqrt{221}$.

(4.14) reduces to $b<25 / 4$ which is true. (4.16), on dividing by $b^{2}$ and substituting for $b$, reduces to

$$
\frac{4 d}{b}>\frac{146}{25}+\frac{2 \sqrt{221}}{5} \sqrt{1-\frac{1}{b}} .
$$

This is easily seen to be true since $d / b \geq \sqrt{221 / 60}$ and $b<1.05$, in this case.

LEMMA 24. If $\phi=\rho \phi_{2}$ then again (4.6) is true.

PROOF. Here $\phi=\rho\left(y^{2}-2 z^{2}\right), \rho=\Delta / \sqrt{8}$. Choose $y \equiv y_{0} \quad(\bmod 1)$ such that $1 / 2 \leq|y| \leq 1$ and $z \equiv z_{0} \quad(\bmod 1)$ such that $0 \leq|z| \leq 1 / 2$. have

If $\sqrt{\frac{2}{4}-\frac{1}{4 \rho}}<|y| \leq 1$ or if $0 \leq|z|<\sqrt{\frac{1}{8}+\frac{1}{8 \rho}}$ (which are true if $\rho<1$ ), we

$$
-1 / 4<\rho\left(y^{2}-2 z^{2}\right) \leq \rho<d-1 / 4
$$


proving (4.8) and hence (4.6).

Let now $\rho \geq 1, \frac{1}{2} \leq|y| \leq \sqrt{\left(\frac{2}{4}-\frac{1}{4 \rho}\right)}$ and $\sqrt{\frac{1}{8}+\frac{1}{8 \rho}} \leq|z| \leq \frac{1}{2}$. This is same thing as saying that $\left(y_{0}, z_{0}\right) \equiv\left(\frac{1}{2}+\theta, \frac{1}{2}+\theta^{\prime}\right)(\bmod 1)$ where

$$
|\theta| \leq \sqrt{\left(\frac{2}{4}-\frac{1}{4 \rho}\right)}-\frac{1}{2} \quad \text { and } \quad\left|\theta^{\prime}\right| \leq \frac{1}{2}-\sqrt{\frac{1}{8}+\frac{1}{8 \rho}} .
$$

ClAIM. If (4.6) has no solution in $(x, y, z) \equiv\left(x_{0}, 1 / 2+\theta, 1 / 2+\theta^{\prime}\right) \quad(\bmod 1)$ where $\theta$ and $\theta^{\prime}$ are given as in (4.18) then $h=g=0$.

Assuming the claim, if (4.6) has no solution then one must have

$$
Q=x^{2}+\rho\left(y^{2}-2 z^{2}\right) .
$$

Now if $\rho>1$, let $\rho=1+\delta, \delta>0$; then since $\rho=\Delta / \sqrt{8} \leq \sqrt{6 / 5}$, we have

$$
0<Q(3,0,2)=9-8 \rho=1-8 \delta<1-\varepsilon
$$

on taking $\varepsilon$ sufficiently small. This contradicts (4.4). If $\rho=1, Q(1,1,1)=0$, so that $Q$ is a zero form, contradicting the hypothesis of Theorem 2 . Hence there is no $Q$ for which (4.6) has no solution. This proves the lemma.

PROOF OF ClAIM. If (4.6) has no solution, for all integers $p, q, r$ we must have either

$$
\begin{aligned}
& Q\left(p+x_{0}, q+\frac{1}{2}+\theta, r+\frac{1}{2}+\theta^{\prime}\right) \geq d, \quad \text { or } \\
& Q\left(p+x_{0}, q+\frac{1}{2}+\theta, r+\frac{1}{2}+\theta^{\prime}\right) \leq 0 .
\end{aligned}
$$

Take $q=-1, r=0$ and choose an integer $p$ such that

$$
1 \leq \alpha=\left|p+x_{0}+h\left(-\frac{1}{2}+\theta\right)+g\left(\frac{1}{2}+\theta^{\prime}\right)\right| \leq 3 / 2 \text {. }
$$

Then from (4.19) and (4.18) we have either

(a)

$$
\begin{aligned}
\alpha^{2} & \geq d+2 \rho\left(\frac{1}{2}+\theta^{\prime}\right)^{2}-\rho\left(\frac{1}{2}-\theta\right)^{2} \\
& \geq d+2 \rho\left(\frac{1}{8}+\frac{1}{8 \rho}\right)-\rho\left(\frac{2}{4}-\frac{1}{4 \rho}\right)
\end{aligned}
$$




$$
\begin{aligned}
& =d+\frac{1}{2}-\frac{\rho}{4}=\left(\frac{40}{6}\right)^{1 / 3} \rho^{2 / 3}+\frac{1}{2}-\frac{\rho}{4} \\
& =f(\rho) \quad(\text { say }), \text { or }
\end{aligned}
$$

(b) $\quad \alpha^{2} \leq 2 \rho\left(1-\sqrt{\frac{1}{8}+\frac{1}{8 \rho}}\right)^{2}-\rho\left(1-\sqrt{\frac{2}{4}-\frac{1}{4 \rho}}\right)^{2}$

$$
\begin{aligned}
& =\frac{3}{4} \rho+\frac{2}{4}-\sqrt{2 \rho^{2}+2 \rho}+\sqrt{2 \rho^{2}-\rho} \\
& =g(\rho) \text { (say). }
\end{aligned}
$$

One can easily check that $f(\rho)$ and $g(\rho)$ are increasing functions of $\rho$ for $1 \leq \rho=\Delta / \sqrt{8} \leq \sqrt{6 / 5}$; therefore either $\alpha^{2} \geq f(1)>2.132$ or $\alpha^{2} \leq$ $g(\sqrt{6 / 5})<0.3$, that is, either $\alpha>1.46$ or $\alpha<0.55$. From (4.20) we must have $1.46<\alpha \leq 3 / 2$, that is,

$$
1.46<p+x_{0}+h\left(-\frac{1}{2}+\theta\right)+g\left(\frac{1}{2}+\theta^{\prime}\right)<1.54(\bmod 1)
$$

that is,

(4.21) $\frac{1}{2}-0.04<x_{0}-h\left(\frac{1}{2}-\theta\right)+g\left(\frac{1}{2}+\theta^{\prime}\right)<\frac{1}{2}+0.04(\bmod 1)$.

Similarly taking $(q, r)=(0,-1)$ or $(-1,-1)$ and then choosing the integer $p$ suitably, (4.19) gives

(4.22) $\frac{1}{2}-0.04<x_{0}+h\left(\frac{1}{2}+\theta\right)-g\left(\frac{1}{2}-\theta^{\prime}\right)<\frac{1}{2}+0.04 \quad(\bmod 1)$
(4.23) $\frac{1}{2}-0.04<-x_{0}+h\left(\frac{1}{2}-\theta\right)+g\left(\frac{1}{2}-\theta^{\prime}\right)<\frac{1}{2}+0.04 \quad(\bmod 1)$.

Adding (4.23) to (4.21) and (4.22) respectively we get

$$
\begin{aligned}
& -0.08<g<0.08 \quad(\bmod 1) \\
& -0.08<h<0.08 \quad(\bmod 1) .
\end{aligned}
$$

Since, from (4.3) $|g| \leq 1 / 2,|h| \leq 1 / 2$, we must have

$$
-0.08<h, g<0.08 \text {. }
$$

Let $P=(h, g)$ in the plane $\mathbb{R}^{2}$ and $\mathscr{R}$ be the region

$$
\mathscr{R}=\left\{(x, y) \in \mathbb{R}^{2}-0.08<x, y<0.08\right\} .
$$


Then (4.25) gives $p \in \mathscr{R}$. Let $U=\left(\begin{array}{l}34 \\ 23\end{array}\right)$ be an automorph of $\phi(y, z)=$ $\rho\left(y^{2}-2 z^{2}\right)$. Let $A=(0,0)$, and

$$
U(\mathscr{R})=\left\{(x, y) \in \mathbb{R}^{2}-0.56<x<0.56,-0.4<y<0.4\right\} .
$$

Clearly $U(\mathscr{R}) \cap(\mathscr{R}+B)=\emptyset$ for all $B \in \mathbb{Z}^{2}, B \neq A$. The unimodular transformation $\left(\begin{array}{cc}1 & 0 \\ 0 & U^{n}\end{array}\right)$ for all integers $n \equiv 0$ transforms $Q$ into

$$
Q(X, Y, Z)=\left(X+h_{n} Y+g_{n} Z\right)^{2}+\rho\left(Y^{2}-2 Z^{2}\right) .
$$

The above argument shows that, if (4.6) has no solution then $U^{n}(P)=\left(h_{n}, g_{n}\right)$ must also satisfy (4.24). Therefore by Lemma 18 , we have

$$
U(P)-A=P,
$$

which gives $h=0, g=0$, noting that $U(P)=\left(h_{1}, g_{1}\right)=(3 h+4 g, 2 h+3 g)$. This proves the claim, and hence completes the proof of Theorem 2.

REMARK. One can improve the constant $10 / 3$ in Theorem 2 slightly by this method, but it becomes very difficult to get even the constant $8 / 3$, the second minima for positive values of zero ternary forms.

\section{Acknowledgements}

The author is very grateful to Professor V. C. Dumir and Professor R. J. HansGill for many useful suggestions and discussions during the preparation of this paper.

\section{References}

Bambah, R. P., Dumir, V. C. and Hans-Gill, R. J. (1981), 'Positive values of non-homogeneous indefinite quadratic forms', in: Topics in classical number theory, Colloq. Math. Soc. Jánes Bolyai, (North, Amsterdam), pp. 111-170.

Bambah, R. P., Dumir, V. C. and Hans-Gill, R. J. (1984), 'Positive values of non-homogeneous indefinite quadratic forms II', $J$. Number Theory $18,313-341$.

Barnes, E. S. (1954), 'The inhomogeneous minimum of a ternary quadratic forms', Acta Math. 92, 13-33.

Barnes, E. S. (1955), 'The non negative values of quadratic forms', Proc. London Math Soc. 5(3), 185-196. 
Barnes, E. S. (1956), 'The inhomogeneous minimum of a ternary quadratic forms (II)', Acta Math. 96, 67-97.

Barnes, E. S. (1961), 'The positive values of inhomogeneous ternary quadratic forms', J. Austral. Math. Soc. (Series A) 2, 127-132.

Blaney, H. (1950a), 'Indefinite ternary quadratic forms', Quart.J. Math., Oxford Ser. 1(2), 252-69.

Blaney, H. (1950b), 'Some asymmetric inequalities', Math. Proc. Cambridge Philos. Soc. 46, 359 376.

Davenport, H. (1946), 'Non-homogeneous binary quadratic forms', Proc. Kon. Nederl. Akad. Wetensch. 49, 815-821.

Davenport, H. (1948), 'Non-homogeneous ternary quadratic forms', Acta Math. 80, 65-95.

Dumir, V. C. (1967), 'Asymmetric inequalities for non-homogeneous ternary quadratic forms', Math. Proc. Cambridge Philos. Soc. 63, 291-303.

Dumir, V. C. (1968), 'Positive values of inhomogeneous quadratic forms I', J. Austral. Math. Soc. (Series A) 8, 87-101.

Dumir, V. C. and Hans-Gill, R. J. (1991), 'The second minimum for positive values of nonhomogeneous ternary quadratic forms of type $(1,2)$ ', unpublished.

Grover, V. K. and Raka, M. (1991), 'On inhomogeneous minima of indefinite binary quadratic forms', Acta Math. 167, 287-298.

Macbeath, A. M. (1951), 'A new sequence of minima in the geometry of numbers', Math. Proc. Cambridge Philos. Soc. 47, 266-273.

Margulis, G. A. (1987), 'Indefinite quadratic forms and unipotent flows on homogeneous spaces', Acad. Sci Paris Sér. I Math. 304, 249-253.

Raka, M. (1983), 'On a conjecture of Watson', Math. Proc. Cambridge Philos. Soc. 94, 9-22.

Vulakh, I. Y. (1985), 'On minima of rational indefinite quadratic forms', J. Number Theory 21, 275-285. 